\title{
Static stretching time required to reduce iliacus muscle stiffness
}

$\operatorname{AUTHOR}(\mathrm{S})$ :

Nojiri, Shusuke; Yagi, Masahide; Mizukami, Yu; Ichihashi, Noriaki

\section{CITATION:}

Nojiri, Shusuke ...[et al]. Static stretching time required to reduce iliacus muscle stiffness. Sports Biomechanics 2021, 20(7): 901-910

\section{ISSUE DATE:}

2021

URL:

http://hdl.handle.net/2433/265380

\section{RIGHT:}

This is an Accepted Manuscript of an article published by Taylor \& Francis in 'Sports Biomechanics' on [date of publication], available online: https://www.tandfonline.com/10.1080/14763141.2019.1620321.; The full-text file will be made open to the public on 24 Jun 2020 in accordance with publisher's 'Terms and Conditions for Self-Archiving'.; This is not the published version. Please cite only the published version. この論文は出版社版でありません。引用の際には 出版社版をご確認ご利用ください。 


\section{Static stretching time required to reduce iliacus muscle stiffness}

Shusuke Nojiri ${ }^{\mathrm{a}}$, Masahide Yagi ${ }^{\mathrm{a}}$, Yu Mizukami ${ }^{\mathrm{a}}$, Noriaki Ichihashi ${ }^{\mathrm{a}}$

${ }^{a}$ Human Health Sciences, Graduate School of Medicine, Kyoto University

53 Shogoin-Kawahara-cho, Sakyo-ku, Kyoto, 606-8507, Japan

\section{Corresponding Author}

Shusuke Nojiri

E-mail: nojiri.shusuke.35v@st.kyoto-u.ac.jp

Human Health Sciences, Graduate School of Medicine, Kyoto University

53 Shogoin-Kawahara-cho, Sakyo-ku, Kyoto, 606-8507, Japan 


\section{Static stretching time required to reduce iliacus muscle stiffness}

Static stretching (SS) is an effective intervention to reduce muscle stiffness and is also

5 performed for the iliopsoas muscle. The iliopsoas muscle consists of the iliacus and

6 psoas major muscles, among which the former has a greater physiological cross-

7 sectional area and hip flexion moment arm. Static stretching time required to reduce

8 muscle stiffness can differ among muscles, and the required time for the iliacus muscle

9 remains unclear. The purpose of this study was to investigate the time required to reduce

10 iliacus muscle stiffness. Twenty-six healthy men participated in this study. A 1-min hip

11 extension SS was performed five times. Shear elastic modulus, an index of muscle

12 stiffness, of the iliacus muscle was measured using ultrasonic shear wave elastography

13 before SS and immediately after each SS. One-way repeated analysis of variance

14 showed a statistical effect of time on the shear elastic modulus. A paired $t$-test with

15 Holm adjustment revealed that the shear elastic moduli after 1-5 SS were statistically

16 lower than that before SS. In addition, the shear elastic modulus after 5 SS was

17 statistically lower than that after 1 SS. The results suggested that the stiffness of the

18 iliacus muscle decreased with 1-min SS and further decreased with 5-min SS. 
19 (200 words)

20

21 KEYWORDS

22 Iliacus muscle

$23 \quad$ Static stretching

24 Ultrasonic shear wave elastography 
Limited hip extension range of motion (ROM) owing to increased stiffness or shortening of the

iliopsoas muscle is one of the functional impairments observed in athletes and patients (Ferber,

Kendall, \& McElroy, 2010; Harvey, 1998; Roach et al., 2015). Limited hip extension ROM can

be a risk factor for various musculoskeletal disorders (Delp, Hess, Hungerford, \& Jones, 1999;

Krivickas \& Feinberg, 1996). Limited hip extension ROM reduces peak hip extension angle during gait (Tsukagoshi et al., 2015), which leads to changes in gait such as shortened step length, decreased gait velocity, and increased pelvic motion (Kerrigan, Lee, Collins, Riley, \& Lipsitz, 2001; Miki et al., 2004; Perron, Malouin, Moffet, \& McFadyen, 2000).

The iliopsoas muscle consists of the iliacus and psoas major muscles. The iliacus muscle has greater physiological cross-sectional area (PCSA) and hip flexion moment arm than the psoas major muscle (Blemker \& Delp, 2005; Klein Horsman, Koopman, van der Helm, Prosé, \& Veeger, 2007). Therefore, increased stiffness or shortening of the iliacus muscle affects hip extension ROM more strongly than similar changes in the psoas major muscle. previous studies have used ROM (Boyce \& Brosky, 2008; Ryan et al., 2008), passive torque, and passive stiffness (Fowles, Sale, \& MacDougall, 2000; S. Peter Magnusson, Simonsen, Aagaard, 
44 stiffness because it is influenced by not only muscle stiffness but also pain and stretch tolerance

45 (Weppler \& Magnusson, 2010). Passive torque and passive stiffness reflect the stiffness of many

46 tissues other than the muscle (e.g., ligaments and joint capsule).

Recently, shear elastic modulus, assessed using ultrasonic shear wave elastography

(SWE), has been used as an index of muscle stiffness (Kusano et al., 2017; Umegaki et al., 2015;

Umehara et al., 2017). SWE estimates muscle stiffness by calculating shear elastic modulus from

shear wave speed (Bercoff, Tanter, \& Fink, 2004). Several studies reported a high correlation

between the shear elastic modulus and passive muscle force (Eby et al., 2013; Koo, Guo, Cohen,

\& Parker, 2013). Therefore, the stiffness of an individual muscle can be evaluated using SWE.

effective stretching, and is useful in time-limited situations such as clinical and athletic situations.

A few studies have investigated the time required to reduce muscle stiffness and reported different

results (Kusano et al., 2017; Nakamura, Ikezoe, Takeno, \& Ichihashi, 2013). One of the potential

reasons for the different results could be the innate differences in the targeted muscles, especially

muscle size. With regard to muscle size, the iliacus muscle has a much smaller volume compared

to the hamstring muscles or the gastrocnemius (Klein Horsman et al., 2007). Therefore, if the time 
62 the gastrocnemius. In addition, it was reported that passive torque decreased gradually even after

63 a statistically significant reduction in passive torque occurred compared with before SS

64 (Nakamura et al., 2013). Therefore, it is also important to investigate the time course of muscle

65 stiffness after the first statistical difference is observed to perform effective SS.

66 Thus far, no study has investigated the effect of SS on the iliacus muscle. While several studies have performed a long-term intervention by using hip extension SS (Kerrigan, Xenopoulos-Oddsson, Sullivan, Lelas, \& Riley, 2003; Watt et al., 2011), its effect on muscle stiffness or the time course remains unclear.

SS to reduce the stiffness of the iliacus muscle. We hypothesised that the time required to reduce muscle stiffness of the iliacus muscle would be shorter than that of the hamstring muscles or the gastrocnemius reported in previous studies.

\section{Methods}

\section{Participants}

The sample size required for multiple comparisons after a one-way repeated analysis of variance

$($ ANOVA $)($ effect size $=0.58, \alpha$ error $=0.05$, and power $=0.80)$ was calculated using $\mathrm{G}^{*}$ power 
based on a previous study that investigated the acute effect of SS using SWE (Kusano et al., 2017).

The calculated sample size was 26 . Twenty-six men (age: $23.2 \pm 2.9$ years; height: $170.5 \pm 5.9$

$\mathrm{cm}$; mass: $63.7 \pm 6.3 \mathrm{~kg}$ ) were recruited for this study. None of the participants had

musculoskeletal injury or neuromuscular disease in the hip or lumbar region. The exclusion

criteria were (1) difficulty in taking the position at which the shear elastic modulus was measured

owing to limited hip extension ROM, (2) no stretch sensation in their upper leg at maximal hip

extension, and (3) pain or numbness in the right leg during SS.

School and the Faculty of Medicine (R0233-3). Each participant provided written informed consent for participation in the study.

\section{Experimental protocol}

92 Hip extension SS was performed for $1 \mathrm{~min}$; this was repeated five times with 1-min rest

93 intervals, corresponding to the time for measurement of shear elastic modulus. We used 1 min of

94 SS to test the hypothesis that the time required to reduce the iliacus muscle stiffness would be

95 shorter than $2 \mathrm{~min}$. Also, we performed a total of 5 min of SS based on a previous study

96 (Nakamura et al., 2013). The shear elastic modulus of the iliacus muscle was measured before

97 SS (bSS) and immediately after each round of SS (SS1-SS5), corresponding to a total of six 
98

99

100

101

measurements.

The participants were instructed to relax and not to activate their lower limb muscles

throughout the experiment. Each participant lay supine with the hip joint positioned at the edge of the bed. The left hip was passively flexed as much as possible to tilt the pelvis backward maximally by an investigator (YM), and thereafter, the pelvis was fixed to the bed with a nonelastic belt. The right hip was held at $5^{\circ}$ extension by another investigator (SN) and the shear elastic modulus was measured (Figure 1). All six measurements of the shear elastic modulus were performed at this position. We confirmed via a preliminary experiment that the shear elastic modulus of the iliacus muscle did not decrease by maintaining this position for $1 \mathrm{~min}$. In hip extension SS, the left hip was maintained at maximal flexion by an investigator (YM), and the right hip was extended by another investigator (SN) to the maximal angle that could be achieved without the participants feeling any discomfort or pain (Figure 2). The right knee was maintained in full extension to avoid elongation of the rectus femoris. The maximal hip extension angle was measured during each round of SS and after all rounds of SS, using a $1^{\circ}$ scale goniometer. The hip extension angle was defined as the angle between the trunk and the femur. All measurements were obtained by the same three examiners, one of whom (MY) performed the measurement of the shear elastic modulus and the hip extension angle, and two of whom (YM and SN) fixed the limb position. 


\section{Measurement of shear elastic modulus}

118 Shear elastic modulus was measured to assess the muscle stiffness. Ultrasonic SWE (Aixplorer;

119 SuperSonicImagine, Aix-en-Provence, France) with a SuperLinear SL 10-2 probe was used to

measure the shear elastic modulus. The shear elastic modulus of the iliacus muscle was measured

in the right limb. The measurement site was defined as a level $4 \mathrm{~cm}$ distal from anterior superior

iliac spines, because it was reported that the iliopsoas muscle was located most superficially at

this level (Jiroumaru, Kurihara, \& Isaka, 2014). The iliacus muscle belly was identified at this

level using a B-mode ultrasonic image. Subsequently, the measurement site was determined and

marked on the skin. The probe was placed parallel to the muscle fiber on the mark, and it was

confirmed that the muscle fiber was uninterrupted on the ultrasonic image. Subsequently, the time required for the two measurements in each round was $<1 \mathrm{~min}$. muscle belly. A circle was drawn in full size within the ROI. The mean shear wave speed in the 


$$
\mathrm{G}(\mathrm{kPa})=\rho \mathrm{V}^{2},
$$

where $\rho$ is the muscle mass density, which is assumed to be $1000 \mathrm{~kg} / \mathrm{m}^{3}$ (Gennisson, Cornu,

Catheline, Fink, \& Portero, 2005). The calculation of shear elastic modulus values was performed

by an investigator (SN), who was different from the investigator who measured the shear elastic modulus.

Fleiss (1979) for the two measurements at bSS as an index of the reliability of shear elastic modulus values. ICC1,1 was 0.85 (95\% confidence interval [CI]: $0.69-0.93)$, and ICC1,2 was 0.92 (95\% CI: 0.82-0.96), and therefore good reliability was observed (Portney \& Watkins, 2000; Shrout \& Fleiss, 1979).

\section{Statistical analysis}

146 Statistical analysis was performed using SPSS Statistics (version 22; IBM, Armonk, NY, USA).

147 A one-way repeated measures ANOVA was performed to assess the effect of time on the shear

148 elastic modulus. When a statistical effect was observed, a post hoc test was performed. A paired $149 t$-test was performed between the shear elastic modulus at bSS and that at SS1-SS5.

150 Furthermore, the shear elastic moduli were compared between the time when the first statistical difference compared with bSS was observed and afterward, by using a paired $t$-test. The level of 
152 statistical rareness was set at $P<0.05$. In post hoc tests, $P$ values were corrected with Holm

153 adjustment in each $t$-test. We estimated the effect size using partial $\eta^{2}$ and $\mathrm{r}$ for the one-way

154 repeated measures ANOVA and post hoc test, respectively. The partial $\eta^{2}$ value is considered

155 moderate and large when it is $\geq 0.07$ and $\geq 0.14$, respectively (Cohen, 1988).

156

Results

The shear elastic modulus at each time point is shown in Table 1 as a mean \pm standard deviation.

The maximal hip extension angle during each round of SS is shown in Table 2 as a mean \pm standard deviation. statistically lower than at bSS. Moreover, from a comparison of the shear elastic moduli using a paired t-test between SS1 and SS2-SS5, the shear elastic modulus at SS5 was observed to be statistically lower than at SS1.

\section{Discussion and implications}

In this study, we investigated the effect of hip extension SS on the stiffness of the iliacus muscle using SWE. The shear elastic moduli at measurements SS1-SS5 were statistically lower than that 
at bSS. This result suggests that the stiffness of the iliacus muscle decreased with 1 min of SS,

and is consistent with our hypothesis. Furthermore, the shear elastic modulus at SS5 was

statistically lower than that at SS1. This result suggests that the stiffness of the iliacus muscle

further decreased with 5 min of SS compared with 1 min of SS. To the best of our knowledge,

this is the first study to demonstrate the time required for hip extension SS to reduce the stiffness

of the iliacus muscle.

Previous studies reported that passive torque or passive stiffness decreased after 2-2.5

min of SS (Nakamura et al., 2013; Nordez, Cornu, \& McNair, 2006) and did not decrease after

1-1.5 min of SS (S. P. Magnusson, Aagard, Simonsen, \& Bojsen-Møller, 1998; McNair,

Dombroski, Hewson, \& Stanley, 2001). Therefore, more than 2 min of SS has been considered

necessary to reduce muscle stiffness (Akagi \& Takahashi, 2013; Nakamura et al., 2014, 2013).

However, the shear elastic modulus of the iliacus muscle decreased after 1 min of SS in this study.

The reasons for the shorter time in this study could be explained by the difference in the muscle size and the index of muscle flexibility.

(S. P. Magnusson et al., 1998; Nordez et al., 2006) or the gastrocnemius (McNair et al., 2001; 
188 the shorter time required. With regards to muscle size, the volume of the iliacus muscle is smaller than that of the hamstring muscles or the gastrocnemius (Klein Horsman et al., 2007). Therefore, the shorter time in this study could be explained by the smaller size of the iliacus muscle compared with that of hamstring or the gastrocnemius muscles. The difference in the index of muscle stiffness could also be the reason for the shorter time required in the current study. The referred studies used passive torque or passive stiffness as an index of muscle stiffness (S. P. Magnusson et al., 1998; McNair et al., 2001; Nakamura et al., 2013; Nordez et al., 2006). While those indices reflect the stiffness of not only the muscle but also the entire joint complex, we evaluated the stiffness of the iliacus muscle solely by using SWE. By using shear elastic modulus as an index of muscle stiffness, Kusano et al. (2017) reported much shorter time than the referred studies that used passive torque and passive stiffness as an index of muscle stiffness (S. P. Magnusson et al., 1998; McNair et al., 2001; Nakamura et al., 2013; Nordez et al., 2006). In other words, it is indicated that the stiffness of muscle decreases earlier than that of the entire joint complex. every SS and a statistically significant difference was observed with SS5 compared with SS1. This result suggests that the stiffness of the iliacus muscle decreased further with 5 min of SS than 
206 minute during 5 min of SS, which was similar to the result of this study. They showed that passive

207 torque decreased statistically after 2 min of SS compared with before SS and decreased

208 statistically after 5 min of SS compared with 2 min of SS. The mechanism of gradual decrease of

209 passive torque was reported to be viscoelastic stress relaxation, which is a decline in the stress or

210 force of the tissues when held at an extended position (Taylor, Dalton, Seaber, \& Garrett, 1990).

211 It has been reported that the force declines rapidly in the first few tens of seconds and thereafter

212 declines gradually until 5 min (McNair et al., 2001; Toft, Sinkjaer, Kålund, \& Espersen, 1989). In this study, five repetitions of SS could cause viscoelastic stress relaxation as well as 5 consecutive min of SS in the previous study (Nakamura et al., 2013).

In this study, a gradual decrease in muscle stiffness similar to that in consecutive SS was observed in repeated SS. This result could be clinically beneficial. This is because repeating 1 min of SS five times may be much easier for therapists than performing 5 consecutive min of SS. required to reduce the shear elastic modulus of the iliacus muscle because the effect of SS shorter than $1 \mathrm{~min}$ is unclear. However, we confirmed that the shear elastic modulus of the iliacus muscle hardly decreased in a preliminary experiment in which $30 \mathrm{~s}$ of SS was repeated. Therefore, we chose to repeat $1 \mathrm{~min}$ of SS. Second, we investigated only the acute effect of SS, and the duration 
224 intervals (i.e., 30-60 s), the effect of long-term intervention, and the effect on performance will

225 be further investigated. Third, the effects of SS on the psoas major remain unclear, although we

226 chose the iliacus muscle rather than the psoas major, based on the greater PCSA and hip flexion

227 moment arm.

\section{Conclusion}

230 In this study it was suggested that the stiffness of the iliacus muscle decreased with 1 min of hip extension SS and further decreased with 5 min of SS. 
234 This work was supported by Grant-in-Aid for Scientific Research (B) 15H03043. We would like

235 to thank Ms. Ibuki and Editage (www.editage.jp) for English language editing.

237 Disclosure statement

238 The authors declare no conflicts of interest.

\section{$240 \quad$ Funding}

241 This work was supported by Grant-in-Aid for Scientific Research (B) (15H03043).

\section{References}

244 Akagi, R., \& Takahashi, H. (2013). Acute effect of static stretching on hardness of the gastrocnemius muscle. Medicine and Science in Sports and Exercise, 45(7), 1348-1354. https://doi.org/10.1249/MSS.0b013e3182850e17

247 Bercoff, J., Tanter, M., \& Fink, M. (2004). Supersonic shear imaging: a new technique for soft tissue elasticity mapping. IEEE Transactions on Ultrasonics, Ferroelectrics, and Frequency Control, 51(4), 396-409. https://doi.org/10.1109/TUFFC.2004.1295425 
architectures and geometries. Annals of Biomedical Engineering, 33(5), 661-673.

Boyce, D., \& Brosky, J. A. (2008). Determining the minimal number of cyclic passive stretch repetitions recommended for an acute increase in an indirect measure of hamstring length. Physiotherapy Theory and Practice, 24(2), 113-120. https://doi.org/10.1080/09593980701378298

Cohen, J. (1988). Statistical power analysis for the behavioral sciences (2nd ed). New York: Lawrence Erlbaum Associates.

Delp, S. L., Hess, W. E., Hungerford, D. S., \& Jones, L. C. (1999). Variation of rotation moment arms with hip flexion. Journal of Biomechanics, 32(5), 493-501. https://doi.org/10.1016/S0021-9290(99)00032-9

Eby, S. F., Song, P., Chen, S., Chen, Q., Greenleaf, J. F., \& An, K.-N. (2013). Validation of shear wave elastography in skeletal muscle. Journal of Biomechanics, 46(14), 2381-2387. https://doi.org/10.1016/j.jbiomech.2013.07.033

Ferber, R., Kendall, K. D., \& McElroy, L. (2010). Normative and critical criteria for iliotibial band and iliopsoas muscle flexibility. Journal of Athletic Training, 45(4), 344-348. https://doi.org/10.4085/1062-6050-45.4.344 
of the human plantarflexors. Journal of Applied Physiology (Bethesda, Md. : 1985), 89(3),

Gennisson, J. L., Cornu, C., Catheline, S., Fink, M., \& Portero, P. (2005). Human muscle hardness assessment during incremental isometric contraction using transient elastography. Journal of Biomechanics, 38(7), 1543-1550. https://doi.org/10.1016/j.jbiomech.2004.07.013

Harvey, D. (1998). Assessment of the flexibility of elite athletes using the modified Thomas test. British Journal of Sports Medicine, 32(1), 68-70. https://doi.org/10.1136/bjsm.32.1.68

Jiroumaru, T., Kurihara, T., \& Isaka, T. (2014). Establishment of a recording method for surface electromyography in the iliopsoas muscle. Journal of Electromyography and Kinesiology: Official Journal of the International Society of Electrophysiological Kinesiology, 24(4), 445-451. https://doi.org/10.1016/j.jelekin.2014.02.007

Kerrigan, D. C., Lee, L. W., Collins, J. J., Riley, P. O., \& Lipsitz, L. A. (2001). Reduced hip extension during walking: healthy elderly and fallers versus young adults. Archives of Physical Medicine and Rehabilitation, 82(1), 26-30. https://doi.org/10.1053/apmr.2001.18584 
Effect of a hip flexor-stretching program on gait in the elderly. Archives of Physical

Klein Horsman, M. D., Koopman, H. F. J. M., van der Helm, F. C. T., Prosé, L. P., \& Veeger, https://doi.org/10.1016/j.clinbiomech.2006.10.003

Koo, T. K., Guo, J.-Y., Cohen, J. H., \& Parker, K. J. (2013). Relationship between shear elastic 2053-2059. https://doi.org/10.1016/j.jbiomech.2013.05.016

Krivickas, L. S., \& Feinberg, J. H. (1996). Lower extremity injuries in college athletes: relation 9993(96)90137-9 1788. https://doi.org/10.1016/j.jse.2017.04.018 
evaluation of cyclic and static stretch in human skeletal muscle. International Journal of

Magnusson, S. P., Simonsen, E. B., Aagaard, P., \& Kjaer, M. (1996). Biomechanical responses

McNair, P. J., Dombroski, E. W., Hewson, D. J., \& Stanley, S. N. (2001). Stretching at the ankle joint: viscoelastic responses to holds and continuous passive motion. Medicine and Science in Sports and Exercise, 33(3), 354-358. https://doi.org/10.1097/00005768200103000-00003

Miki, H., Sugano, N., Hagio, K., Nishii, T., Kawakami, H., Kakimoto, A., .. Yoshikawa, H. https://doi.org/10.1016/j.jbiomech.2003.09.009

Nakamura, M., Ikezoe, T., Kobayashi, T., Umegaki, H., Takeno, Y., Nishishita, S., \& Ichihashi, N. (2014). Acute effects of static stretching on muscle hardness of the medial Ultrasound in Medicine \& Biology, 40(9), 1991-1997. https://doi.org/10.1016/j.ultrasmedbio.2014.03.024 
Nakamura, M., Ikezoe, T., Takeno, Y., \& Ichihashi, N. (2013). Time course of changes in passive properties of the gastrocnemius muscle-tendon unit during 5 min of static stretching. Manual Therapy, 18(3), 211-215. https://doi.org/10.1016/j.math.2012.09.010

Nordez, A., Cornu, C., \& McNair, P. (2006). Acute effects of static stretching on passive stiffness of the hamstring muscles calculated using different mathematical models. Clinical Biomechanics (Bristol, Avon), 21(7), 755-760. https://doi.org/10.1016/j.clinbiomech.2006.03.005

Perron, M., Malouin, F., Moffet, H., \& McFadyen, B. J. (2000). Three-dimensional gait analysis in women with a total hip arthroplasty. Clinical Biomechanics (Bristol, Avon), 15(7), 504515. https://doi.org/10.1016/S0268-0033(00)00002-4

Portney, L., \& Watkins, M. (2000). Foundations of clinical research: application to practice (2nd ed). New Jersey: Prentice Hall Health.

Roach, S. M., San Juan, J. G., Suprak, D. N., Lyda, M., Bies, A. J., \& Boydston, C. R. (2015). Passive hip range of motion is reduced in active subjects with chronic low back pain compared to controls. International Journal of Sports Physical Therapy, 10(1), 13-20.

Retrieved from http://www.pubmedcentral.nih.gov/articlerender.fcgi?artid=4325283\&tool=pmcentrez\&re ndertype $=$ abstract 
Ryan, E. D., Beck, T. W., Herda, T. J., Hull, H. R., Hartman, M. J., Stout, J. R., \& Cramer, J. T. (2008). Do practical durations of stretching alter muscle strength? A dose-response study. Medicine and Science in Sports and Exercise, 40(8), 1529-1537. https://doi.org/10.1249/MSS.0b013e31817242eb

Shrout, P. E., \& Fleiss, J. L. (1979). Intraclass correlations: uses in assessing rater reliability. Psychological Bulletin, 86(2), 420-428. Retrieved from http://rokwa.x-y.net/Shrout-FleissICC.pdf

Taylor, D. C., Dalton, J. D., Seaber, A. V., \& Garrett, W. E. (1990). Viscoelastic properties of muscle-tendon units. The biomechanical effects of stretching. The American Journal of Sports Medicine, 18(3), 300-309. https://doi.org/10.1177/036354659001800314

Toft, E., Sinkjaer, T., Kålund, S., \& Espersen, G. T. (1989). Biomechanical properties of the human ankle in relation to passive stretch. Journal of Biomechanics, 22(11-12), 11291132. https://doi.org/10.1016/0021-9290(89)90214-5

Tsukagoshi, R., Tateuchi, H., Fukumoto, Y., Akiyama, H., So, K., Kuroda, Y., ... Ichihashi, N. (2015). Factors associated with restricted hip extension during gait in women after total hip arthroplasty. Hip International : The Journal of Clinical and Experimental Research on Hip Pathology and Therapy, 25(6), 543-548. https://doi.org/10.5301/hipint.5000286 
N. (2015). Acute effects of static stretching on the hamstrings using shear elastic modulus hamstring muscle components. Manual Therapy, 20(4), 610-613. https://doi.org/10.1016/j.math.2015.02.006

Umehara, J., Hasegawa, S., Nakamura, M., Nishishita, S., Umegaki, H., Tanaka, H., ... Ichihashi, N. (2017). Effect of scapular stabilization during cross-body stretch on the https://doi.org/10.1016/j.math.2016.10.004 https://doi.org/10.1016/j.pmrj.2010.11.012

Watt, J. R., Jackson, K., Franz, J. R., Dicharry, J., Evans, J., \& Kerrigan, D. C. (2011). Effect of a supervised hip flexor stretching program on gait in elderly individuals. $P M \& R$ : The Journal of Injury, Function, and Rehabilitation, 3(4), 324-329.

Weppler, C. H., \& Magnusson, S. P. (2010). Increasing muscle extensibility: a matter of increasing length or modifying sensation? Physical Therapy, 90(3), 438-449. https://doi.org/10.2522/ptj.20090012 
Table 1 Shear elastic modulus of the iliacus muscle at each time point

\begin{tabular}{ccccccc}
\hline & Shear elastic & \multicolumn{2}{c}{ Vs. bSS } & & \multicolumn{2}{c}{ Vs. SS1 } \\
\cline { 6 - 7 } & modulus $(\mathrm{kPa})$ & $P$ value & effect size $(\mathrm{r})$ & & $P$ value & effect size (r) \\
\hline bSS & $22.1 \pm 3.5$ & - & - & & - & - \\
SS1 & $20.5 \pm 4.2$ & 0.008 & 0.50 & & - & - \\
SS2 & $20.1 \pm 4.4$ & 0.008 & 0.54 & & 0.49 & 0.14 \\
SS3 & $19.8 \pm 3.7$ & $<0.001$ & 0.71 & & 0.28 & 0.29 \\
SS4 & $19.4 \pm 3.5$ & $<0.001$ & 0.69 & & 0.19 & 0.36 \\
SS5 & $18.2 \pm 2.4$ & $<0.001$ & 0.85 & & 0.006 & 0.58 \\
\hline
\end{tabular}

The shear elastic modulus is expressed as a mean \pm standard deviation.

SS: static stretching

Table 2 Maximal hip extension angle during each round of SS and after SS

\begin{tabular}{cc}
\hline & Maximal hip extension angle $\left(^{\circ}\right)$ \\
\hline 1st SS & $19 \pm 4$ \\
2nd SS & $21 \pm 5$ \\
3rd SS & $23 \pm 5$ \\
4th SS & $25 \pm 5$ \\
5th SS & $26 \pm 5$ \\
After SS & $26 \pm 6$
\end{tabular}

Results are expressed as a mean \pm standard deviation.

The angle was measured during each round of SS and after all rounds of SS. The angle during 2nd SS was indicated as the maximal angle, which was a result of 1st SS, for example. 

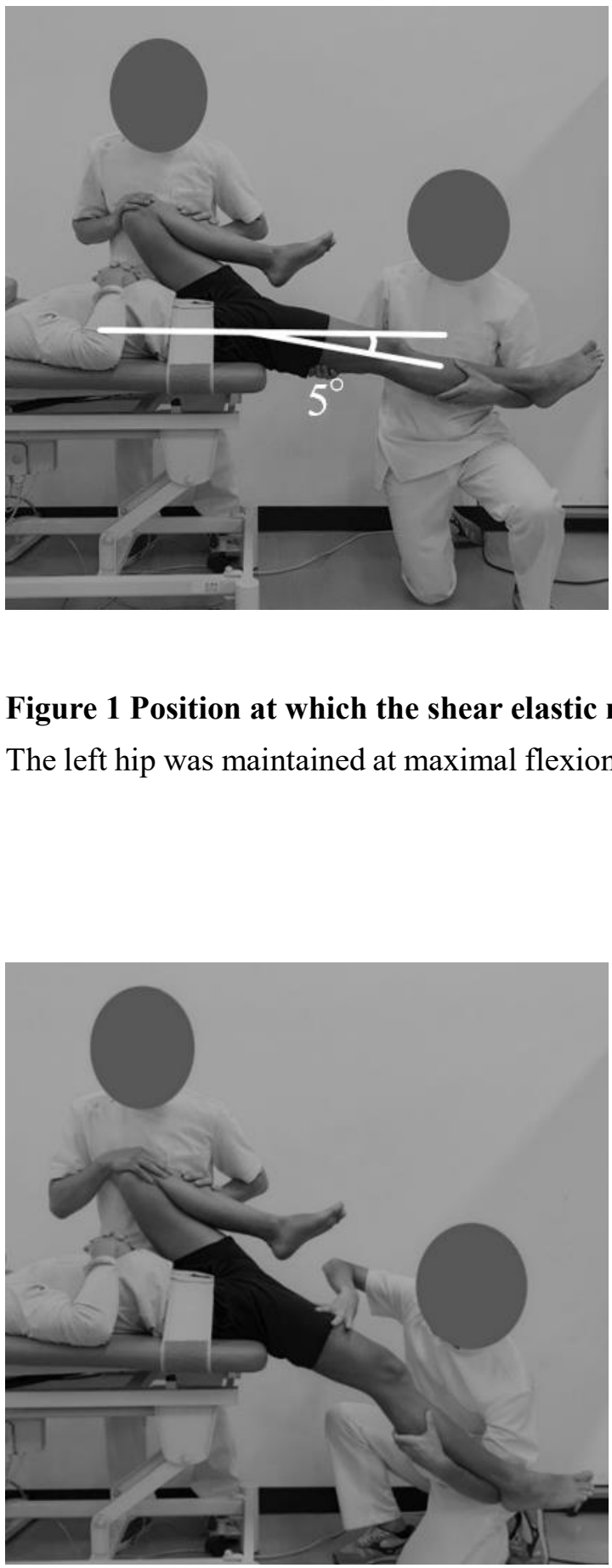

\section{Figure 2 Position of static stretching}

The left hip was maintained at maximal flexion and the right hip was extended to the maximal angle at which there was no pain or discomfort. 


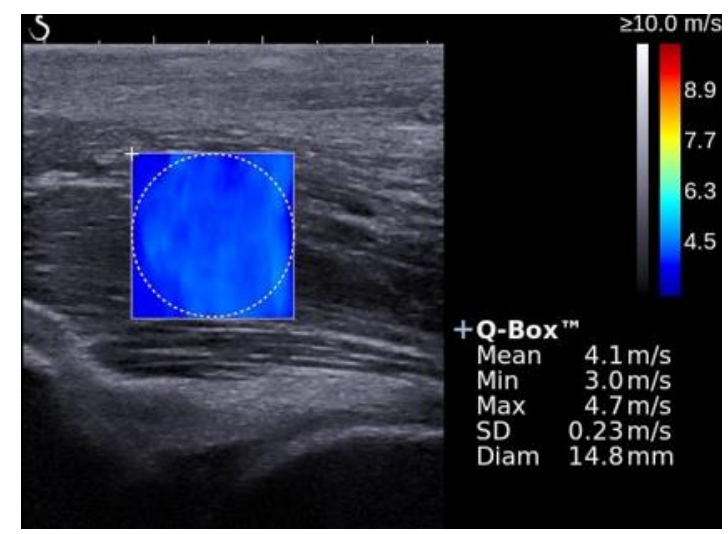

397

Figure 3 Typical example of measuring the shear wave speed

399 An ROI, a square of side $1.5 \mathrm{~cm}$, was set at the center of the iliacus muscle belly. A circle was

400 drawn in full size within the ROI. The mean shear wave speed in the circle was calculated 401 automatically. 